\title{
Protease-activated receptor 4 protects mice from Coxsackievirus B3 and H1N1 influenza A virus infection
}

\author{
Kohei Tatsumi ${ }^{\text {a }}$, Clare M. Schmedes ${ }^{\mathrm{a}}$, E. Reaves Houston ${ }^{\mathrm{a}}$, Emily Butler ${ }^{\mathrm{a}}$, Nigel Mackman ${ }^{\mathrm{a}}$, \\ Silvio Antoniak ${ }^{\mathrm{b}, *}$ \\ ${ }^{a}$ Department of Medicine, Thrombosis and Hemostasis Program, Division of Hematology and Oncology, UNC McAllister Heart Institute, University of North Carolina, \\ Chapel Hill, NC, USA \\ ${ }^{\mathrm{b}}$ Department of Pathology and Laboratory Medicine, UNC McAllister Heart Institute, University of North Carolina at Chapel Hill, Chapel Hill, NC, USA
}

\section{A R T I C L E I N F O}

\section{Keywords:}

Protease-activated receptor 4

Innate immune responses

Myocarditis

Influenza

\begin{abstract}
A B S T R A C T
PAR4 is expressed by a variety of cells, including platelets, cardiac, lung and immune cells. We investigated the contribution of PAR4 to viral infections of the heart and lung. Toll-like receptor (TLR) 3-dependent immune responses were analyzed after co-stimulation of PAR4 in murine bone-marrow derived macrophages, embryonic fibroblasts and embryonic cardiomyocytes. In addition, we analyzed Coxsackievirus B3 (CVB3) or H1N1 influenza A virus (H1N1 IAV) infection of PAR4 ${ }^{-/-}(\triangle \mathrm{PAR} 4)$ and wild-type (WT) mice. Lastly, we investigated the effect of platelet inhibition on H1N1 IAV infection. In vitro experiments revealed that PAR4 stimulation enhances the expression of TLR3-dependent CXCL10 expression and decreases TLR3-dependent NFKB-mediated proinflammatory gene expression. Furthermore, CVB3-infected $\triangle$ PAR4 mice exhibited a decreased anti-viral response and increased viral genomes in the heart leading to more pronounced CVB3 myocarditis compared to WT mice. Similarly, H1N1 IAV-infected $\triangle$ PAR4 mice had increased immune cell numbers and inflammatory mediators in the lung, and increased mortality compared with infected WT controls. The study showed that PAR4 protects mice from viral infections of the heart and lung.
\end{abstract}

\section{Introduction}

Protease-activated receptors (PARs) are a family of 4 G-protein coupled receptors (PAR1-4) [1,2]. Serine proteases and matrix metalloproteinases (MMPs), including immune cell-derived proteases and coagulation proteases, can either activate or inactivate PARs depending on the cell type and post-translational modification of the PAR [3]. For instance, thrombin activates both PAR1 and PAR4 as well as human PAR3. Human platelets express PAR1 and PAR4, whereas PAR4 is the functional thrombin receptor on mouse platelets since they lack PAR1 $[4,5]$. A PAR1 inhibitor is FDA-approved to reduce platelet activation in patients in combination with standard anti-platelet dual therapy [6]. However, chronic PAR1 inhibition can increase the risk of hemorrhagic strokes potentially due to a protective role of PAR1 on vascular integrity [6].

We and others showed that PARs are modulators of antiviral responses to infections with the RNA viruses Coxsackievirus B3 (CVB3) and H1N1 influenza A virus (H1N1 IAV) [3,7-10]. For instance, we and others reported that PAR1 enhances and PAR2 inhibits toll-like receptor (TLR3) dependent anti-viral immune responses [7-9,11,12].
Furthermore, we found that thrombin inhibition reduced immune responses to a TLR3 agonist in mice and increased CVB3 myocarditis $[8,11]$. In line with our findings, a recent study showed that thrombin inhibition reduced TLR3-dependent responses in human bronchial epithelial cells [12].

Importantly, there are some conflicting data regarding the contribution of PARs to the outcome in viral infection of the lung [1]. We observed that PAR1-deficiency increased H1N1 infection of mice [8], whereas another group showed a protection in PAR $1^{-/-}$mice after IAV infection [10]. In addition, we and others found that PAR2 ${ }^{-/-}$mice were protected from H1N1 infection $[9,13]$. However, another group found that PAR2 ${ }^{-/-}$mice exhibited more severe disease after H1N1 IAV infection [14]. The reasons for these opposing data are unclear and are the object of ongoing investigations.

Recent studies have focused on PAR4 as a druggable target to reduce platelet activation and thrombosis $[15,16]$. Like PAR1, many cells other than platelets express PAR4, including cardiac myocytes (CMs) and immune cells $[3,17]$. Interestingly, two studies have suggested that PAR4 antagonism might promote cardiac protection by inhibiting cardiomyocyte apoptosis and pathologic platelet activation in ischemia

\footnotetext{
* Corresponding author at: University of North Carolina at Chapel Hill, 116 Manning Drive, Campus Box 7035, Chapel Hill, NC 27599, USA.

E-mail address: antoniak@email.unc.edu (S. Antoniak).
} 
reperfusion injury $[15,17]$. Furthermore, a recent study showed that repeated administration of a PAR4 inhibitor (pepducin P4pal-10) in wild-type (WT) mice daily between day 2 and 4 after infection resulted in reduced H1N1 IAV infection-mediated lung inflammation and improved survival [18].

In the study here, we used global PAR4 deficient mice, to investigate the role of PAR4 in two different RNA virus infections of the heart and lung.

\section{Methods}

\subsection{Mice}

Adult PAR4 $^{-/-}(\triangle \mathrm{PAR} 4)$ and WT $\left(\mathrm{PAR} 4^{+/+}\right)$mice on the C57Bl/6 background were used for this study [5]. All animal experiments were performed in accordance with the guidelines of the animal care and use committee of the UNC at Chapel Hill and complies with National Institutes of Health guidelines.

\subsection{In vitro experiments}

Bone marrow-derived macrophages (BMM) were generated from WT and $\triangle$ PAR4 mouse bone marrow using the L929-conditioned media method [11]. Mouse embryonic fibroblasts (MEFs) and murine CMs were isolated from WT mouse embryos (E14) as described [7,8]. Male and female mice were used to ensure equally sex distribution within the cell preparations. BMMs, MEFs and CMs were cultured in DMEM (Gibco, Gaithersburg, MD, USA) supplemented with 10\% FBS and 1\% penicillin/streptomycin $[7,11]$. Cells were stimulated under FBS-free conditions with $25 \mu \mathrm{g} / \mathrm{mL}$ of the TLR3 agonist polyinosinic:polycytidylic acid (poly I:C, $\gamma$-radiated; Sigma-Aldrich, St. Louis, Mo, USA) and/or PAR4 agonist peptide (AP, AYPGKF, $150 \mu \mathrm{M}$, Abgent, San Diego, Calif., USA) or thrombin (Thr, $5 \mathrm{nM}$, human $\alpha-$ thrombin; Enzyme Research Laboratories, South Bend, IN, USA).

For splenocyte isolation, WT and $\triangle$ PAR4 spleens were harvested and passed through a $70-\mu \mathrm{m}$ cell strainer to generate a single-cell suspension. RBCs were lysed and the splenocytes washed and resuspended at a density of $2 \times 10^{6}$ cells per well of a 24-well plate in RPMI 1640 with $10 \%$ FBS and $1 \%$ penicillin/streptomycin. Cells were stimulated overnight with PAR4 AP $(150 \mu \mathrm{M})$. The next day, splenocytes were used in a natural killer (NK) cell cytotoxicity assay against YAC-1 target cells as described in detail elsewhere [19].

For CVB3 in vitro infectivity studies, CMs were incubated with CVB3 (Nancy Strain, multiplicity of infection $=1$ ) for $45 \mathrm{~min}$, washed and stimulated with thrombin $(5 \mathrm{nM})$ or PAR4 AP $(150 \mu \mathrm{M})$ for $6 \mathrm{~h}$ under FBS-free conditions [7].

\subsection{Real-time PCR and ELISA}

Cardiac injury was quantified by measuring plasma cardiac troponin-I using an ELISA $[8,20]$. Viral genomes and TNF $\alpha$ mRNA expression were analyzed by real-time PCR [11]. Expression data were normalized to hypoxanthine-guanine phosphoribosyltransferase (HPRT). Interferon $\beta$ (IFN $\beta$ ) and CXCL10, CXCL1, CCL5, MCP1 and TNF $\alpha$ protein levels were analyzed by ELISA as described $[8,11]$. Levels of IgG2a were measured with a mouse IgG2a ELISA kit from (Abcam, Cambridge, UK).

\subsection{Virus infection models}

For the CVB3 myocarditis model, male mice aged 6-8 weeks were infected interperitoneally with $10^{5}$ plaque-forming units of CVB3 (Nancy Strain) in $200 \mu \mathrm{L}$ PBS [8]. Virus was propagated and titered as described [7]. Heart function was measured on conscious mice by echocardiography using a VisualSonics Vevo 2100 ultrasound system as described $[8,20]$.
For the H1N1 IAV infection model, the mouse-adapted strain of influenza A/Puerto Rico/8/1934 (PR/8) was propagated in 10-12 day of embryonated eggs and titered with a hemagglutination unit (HAU) assay [21,22]. WT and $\triangle$ PAR4 mice of both sexes between 8 and 12 weeks were infected intranasally with $0.02 \mathrm{HAU}$ in $50 \mu \mathrm{L}$ PBS as described $[8,21,22]$. To analyze immune cell infiltration and cytokine/ chemokine levels, bronchiolar alveolar lavage fluid (BALF) was collected and processed from infected and uninfected mice by postmortem lavage with $3 \times 900 \mu \mathrm{L}$ ice-cold PBS as described [21,22]. Immune cell and red blood cell (RBC) numbers were determined with a Hemavet 950 (Drew Scientific, Miami Lakes, FL, USA) [21,22]. Mice were euthanized if they had $\geq 25 \%$ loss of initial body weight, as specified in our animal protocol $[21,22]$.

\subsection{Histology}

Lung tissues from WT and $\triangle$ PAR4 mice were fixed in $4 \%$ formalin and paraffin-embedded, cut to $4 \mu \mathrm{m}$ thick sections and stained with $\mathrm{H} \&$ E [22].

\subsection{Platelet inhibition}

To inhibit platelet activation, acetylsalicylic acid (ASA, $0.4 \mathrm{mg} / \mathrm{mL}$, Sigma-Aldrich) or clopidogrel $(0.15 \mathrm{mg} / \mathrm{mL}$, Bristol-Myers Squibb/ Sanofi Pharmaceuticals Partnership Bridgewater, NJ, USA) was given via drinking water (ad lib) 3 days prior and after H1N1 IAV infection $[23,24]$. Arachidonic acid-induced $(2.5 \mathrm{mM})$ or ADP-induced $(10 \mu \mathrm{M})$ platelet activation, measured as integrin $\alpha \operatorname{IIb} \beta 3$ activation, was inhibited in platelets isolated from ASA- or clopidogrel-treated mice, respectively.

\subsection{Statistics}

GraphPad Prism 8.01 (GraphPad Software Inc., La Jolla, CA) was used for statistical analysis. Data are represented as mean \pm SEM. The 2-tailed Student's $t$ test was used for 2-group comparison of normally distributed data. Mann-Whitney test was used for 2-group comparison of not normally distributed data. For multiple-group comparison, normally distributed data were analyzed by 1- or 2-way ANOVA tests and were Bonferroni-corrected for repeated measures over time. Survival rates were analyzed by Kaplan-Meier analysis and the log-rank test was applied to compare the survival distribution between the two groups. $P$ value $\leq 0.05$ was regarded as significant.

\section{Results}

\subsection{PAR4 activation increases CXCL10 expression in BMMs and MEFs}

In previous studies, we showed that TLR3-depdendent CXCL10 expression in response to the dsRNA mimetic poly I:C was enhanced by co-stimulation of PAR1 on macrophages and cardiac fibroblasts $[8,11]$. Here, we investigated if PAR4 activation also enhances poly I:C induced CXCL10 expression in BMMs and MEFs. First, we analyzed poly I:C induction of CXCL10 protein expression in BMMs derived from WT and $\triangle$ PAR4 mice. Poly I:C but not the PAR4 activating protease thrombin induced CXCL10 protein expression in both WT and $\triangle$ PAR4 BMMs (Fig. 1A). Importantly, co-stimulation with poly I:C and thrombin significantly increased CXCL10 protein expression in WT BMMs (Fig. 1A). As expected from our previous study [11], thrombin increased CXCL10 protein expression in poly I:C treated $\triangle$ PAR4 BMMs since these cells express PAR1. However, the CXCL10 expression in $\triangle$ PAR4 BMMs was significant lower compared to co-stimulated WT BMMs (Fig. 1A). In addition, $\triangle$ PAR4 BMMs exhibited significant lower CXCL10 levels compared to WT BMMs when stimulated with poly I:C alone, suggesting that the cells may produce a PAR4 activating protease similarly to our recent study with PAR1 $[8,11]$. To further test if specific PAR4 

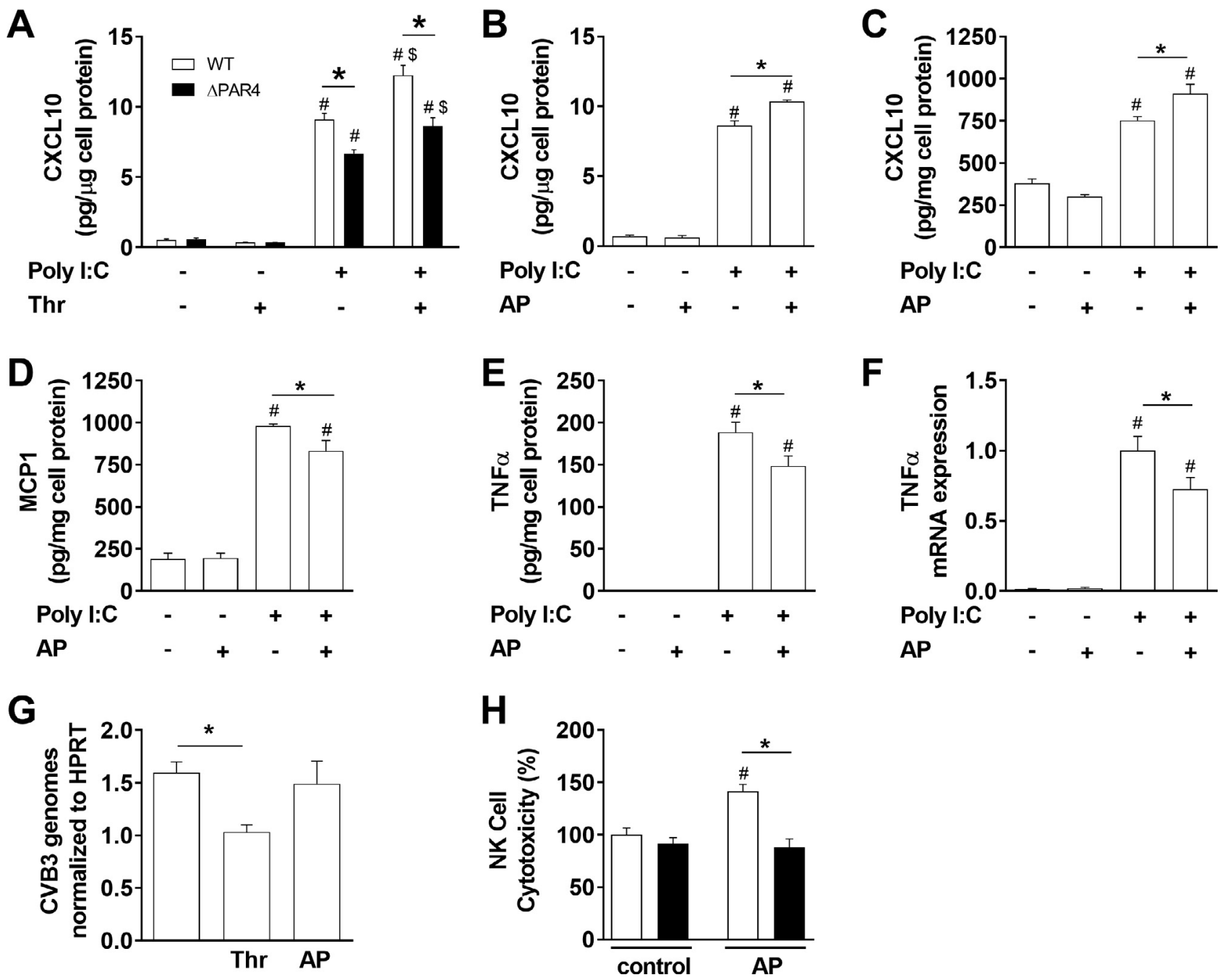

Fig. 1. PAR4 activation modulates innate immune responses in vitro. (A) Levels of CXCL10 in cell culture media of bone marrow-derived macrophages from WT (white bars) and PAR4 ${ }^{-/-}$( $\triangle$ PAR4, black bars) mice in response to the dsRNA mimetic poly I:C $(25 \mu \mathrm{g} / \mathrm{mL})$ and/or thrombin (Thr, $\left.5 \mathrm{nM}\right)$ were analyzed after $7 \mathrm{~h}$ stimulation. WT BMM (B) or WT murine embryonic fibroblasts (C) were stimulated with poly I:C (25 $\mu \mathrm{g} / \mathrm{mL})$ and/or PAR4 activating peptide (AP, $150 \mu \mathrm{M})$ for $7 \mathrm{~h}$ and the CXCL10 protein levels analyzed in cell culture media by ELISA. WT MEF (D, E) were stimulated for $24 \mathrm{~h}$ with poly I:C (25 $\mu \mathrm{g} / \mathrm{mL})$ and/or PAR4 AP (150 $\mu \mathrm{M})$ and the MCP1 and TNF $\alpha$ protein levels were analyzed in the cell culture media by ELISA. (F) TNF $\alpha$ mRNA expression was analyzed by real-time PCR in WT murine CM $4 \mathrm{~h}$ after stimulation with poly I:C $(25 \mu \mathrm{g} / \mathrm{mL})$ and/or PAR4 AP $(150 \mu \mathrm{M})$. Results are shown relative to poly I:C stimulation which was set to 1 . (G) CVB3 genomes were analyzed in WT murine CM by RT-PCR after infection with CVB3 (multiplicity of infection 1) and additional stimulation with thrombin (Thr, $5 \mathrm{nM}$ ) or PAR4 AP $(150 \mu \mathrm{M})$ for $6 \mathrm{~h}$. Genome levels were normalized to HPRT mRNA expression. (H) Natural killer (NK) cell cytotoxicity against YAC-1 cells of splenocytes isolated from WT (white bars) and $\triangle$ PAR4 (black bars) mice after $24 \mathrm{~h}$ stimulation with or without PAR4 AP (150 $\mu \mathrm{M})$. Data (mean \pm SEM; N > 4) were analyzed by 2-way (A, H) and 1-way (B-G) ANOVA. Statistical significance is shown as ${ }^{*} \mathrm{P}<0.05$, \# P $<0.05$ versus unstimulated control within the same genotype or unstimulated control, and $\$ \mathrm{P}<0.05$ versus poly I:C alone.

stimulation increases poly I:C-induced CXCL10 expression in WT BMMs, we used a PAR4 AP. PAR4 AP alone had no effect on CXCL10 expression of WT BMMs (Fig. 1B). Poly I:C increased CXCL10 expression and additional co-stimulation with the PAR4 AP further increased this expression (Fig. 1B). Similar responses of the CXCL10 expression were observed with WT MEFs (Fig. 1C) where poly I:C induced the CXCL10 expression and co-stimulation with PAR4 AP further enhanced the expression of CXCL10 (Fig. 1C).

\subsection{PAR4 activation reduces expression of pro-inflammatory mediators} after poly I:C stimulation of MEFs and CMs

Recently, we reported that PAR1 activation reduces the TLR3-dependent expression of TNFa [1]. To analyze if PAR4 activation produces similar modulation of TLR3 signaling, we analyzed the expression of the proinflammatory chemokine MCP1 and the cytokine TNF $\alpha$ in MEFs and murine CMs after stimulation with PAR4 AP and/or poly I:C.
PAR4 AP alone did not induce MCP1 or TNF $\alpha$ protein expression in WT MEFs (Fig. 1D and E) or TNFa mRNA expression in WT CMs (Fig. 1F). Poly I:C alone led to significantly increased induction of MCP1 and TNF $\alpha$ protein expression in WT MEFs (Fig. 1D and E) and TNF $\alpha$ mRNA expression in WT CMs (Fig. 1F). Interestingly, co-stimulation with poly I:C and PAR4 AP reduced poly I:C induction of both MCP1 and TNF $\alpha$ protein expression in WT MEF and TNF $\alpha$ mRNA expression in WT CMs (Fig. 1D-F).

\subsection{PAR4 activation has no effect on early CVB3 replication in CMs}

CMs, which express PAR4, are the primary site of CVB3 replication in the heart [25]. To analyze if PAR4 activation directly influences CVB3 replication in CMs, WT CMs were infected with CVB3 and stimulated with either thrombin or PAR4 AP. Thrombin but not PAR4 AP stimulation of murine CMs after CVB3 reduced the levels of CVB3 genomes (Fig. 1G) suggesting that PAR1 but not PAR4 reduces CVB3 
A

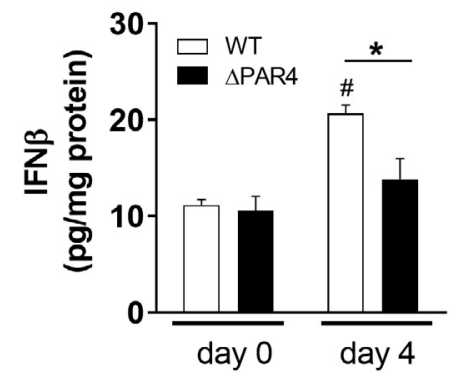

D

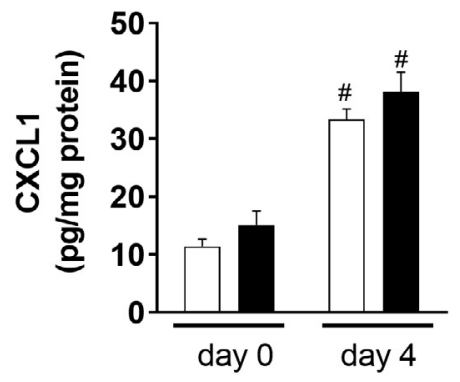

B

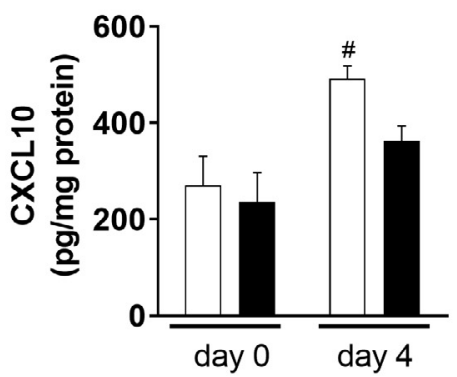

$\mathbf{E}$

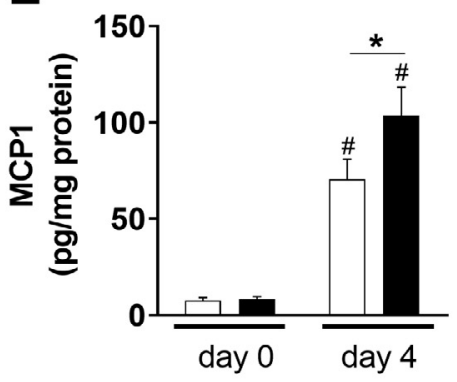

C

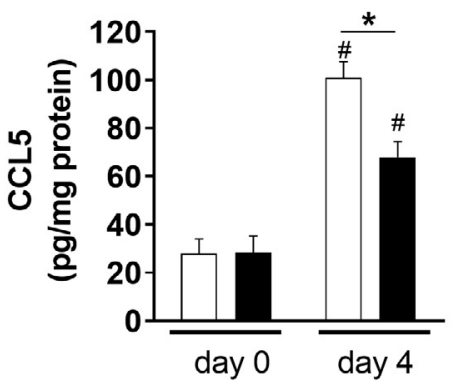

$\mathbf{F}$

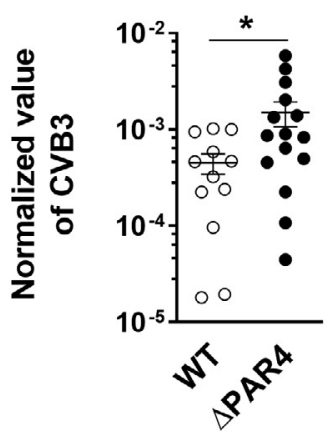

G

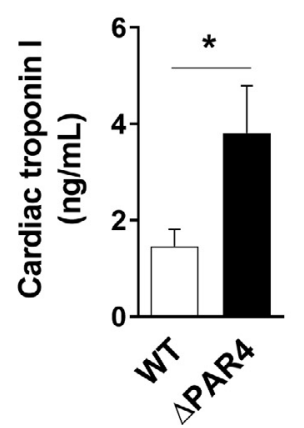

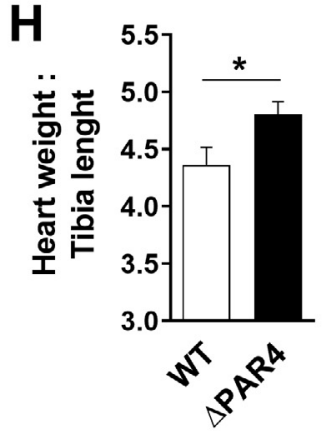

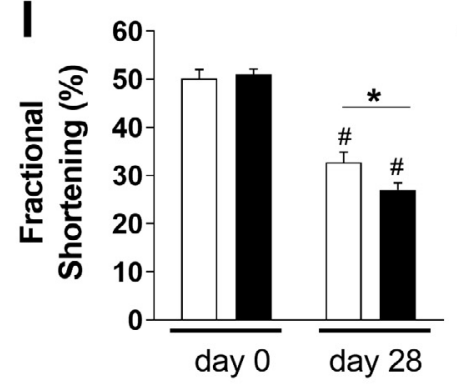

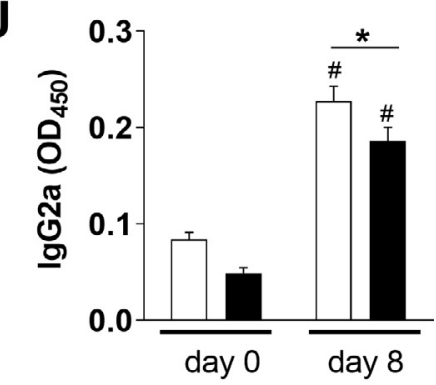

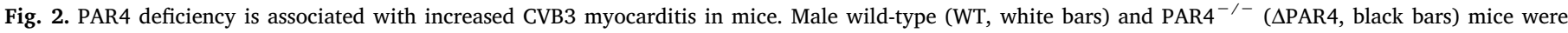

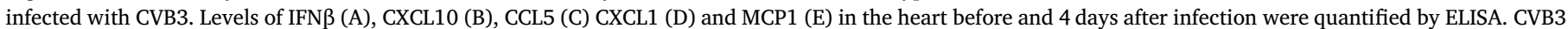

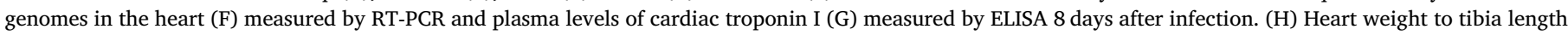

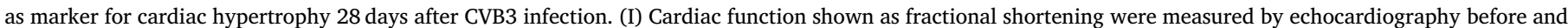

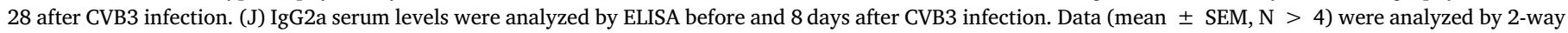
ANOVA (A-E, I and $\mathrm{J}$ ) or by Student's $t$-test $(\mathrm{F}-\mathrm{H}){ }^{*} \mathrm{P}<0.05$, \# $\mathrm{P}<0.05$ versus day 0 within the same genotype.

replication. In line with this, specific PAR1 stimulation with AP reduced CVB3 replication in mouse CMs (unpublished data),

\subsection{PAR4 stimulation increases natural killer cell like activity of splenocytes}

NK cells are one of the first immune cells infiltrating virus-infected tissue, killing infected cells and therefore limiting virus replication. A lack of NK cells was shown to be associated with more a pronounced viral infection [26]. We analyzed the NK cell-like activity of splenocytes isolated from WT and $\triangle$ PAR4 mice. We found that PAR4 AP increased NK cell cytotoxicity of WT but not $\triangle$ PAR4 splenocytes against the target cell YAC-1 (Fig. 1H).

\subsection{PAR4 deficiency is associated with increased CVB3 myocarditis in mice}

To translate our in vitro observations into a human pathologic relevant disease, we first used the CVB3 myocarditis model. Four days after CVB3 infection, hearts of $\triangle$ PAR4 mice had reduced protein levels of IFN $\beta$, and the IFN $\beta$-response genes CXCL10 and CCL5 compared to hearts of WT mice (Fig. 2A-C). Next, we analyzed the expression of the proinflammatory mediators CXCL1 and MCP1 in hearts of WT and $\triangle$ PAR 4 mice before and 4 days after CVB3 infection. We found that MCP1 but not CXCL1 expression was higher in CVB3 infected hearts of $\triangle$ PAR4 mice compared with hearts of WT mice (Fig. 2D and E). Moreover, PAR4-deficiency was associated with higher CVB3 virus genome levels in hearts 8 days after CVB3 infection (Fig. 2F). In addition, the more pronounced cardiac inflammation and the higher virus load was associated with increased cardiac injury, measured by plasma troponin I levels, in $\triangle$ PAR4 compared to WT mice 8 days post infection (p.i.) (Fig. 2G). Increased acute myocarditis in $\triangle$ PAR4 mice resulted in more severe cardiac hypertrophy measured by an increased heart weight to tibia ratio in $\triangle$ PAR4 mice compared to WT mice 28 days p.i. (Fig. $2 \mathrm{H}$ ). Overall, the more severe CVB3 myocarditis in $\triangle$ PAR4 mice resulted in a greater reduction in heart function 28 days p.i. compared to WT mice (Fig. 2I).

\subsection{PAR4 modulates adaptive IgG-dependent immune responses}

PAR4 was shown to be expressed in B lymphocytes [27]. Studies with mice found that increased virus-specific IgGs are detectable from day 7 onwards post CVB3 infection [28]. To analyze if PAR4 has an 
A
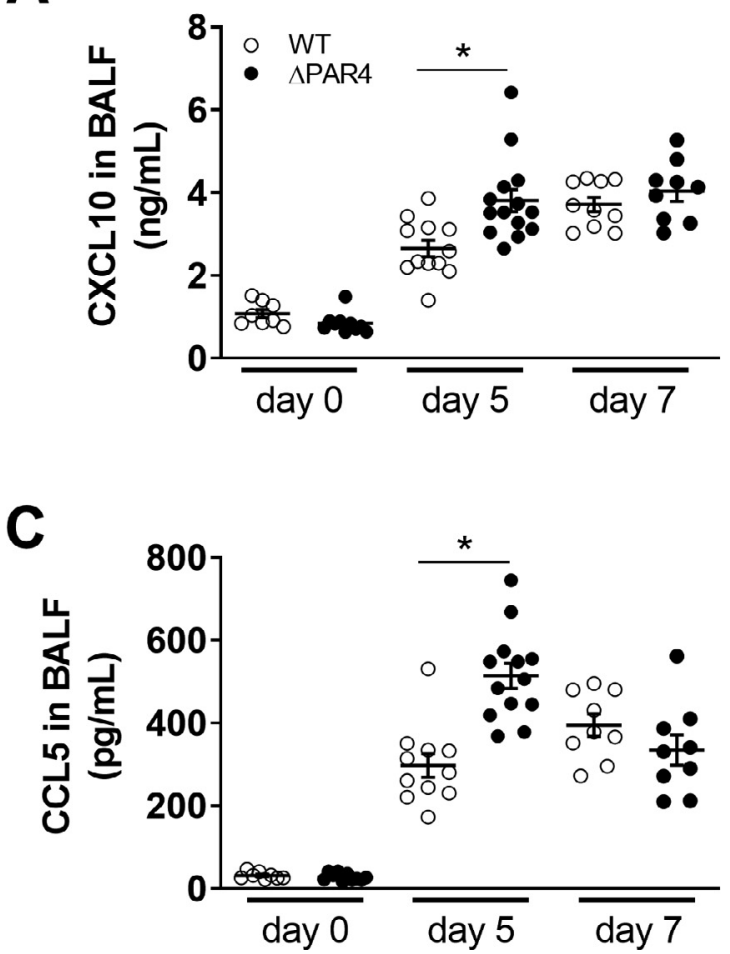

B

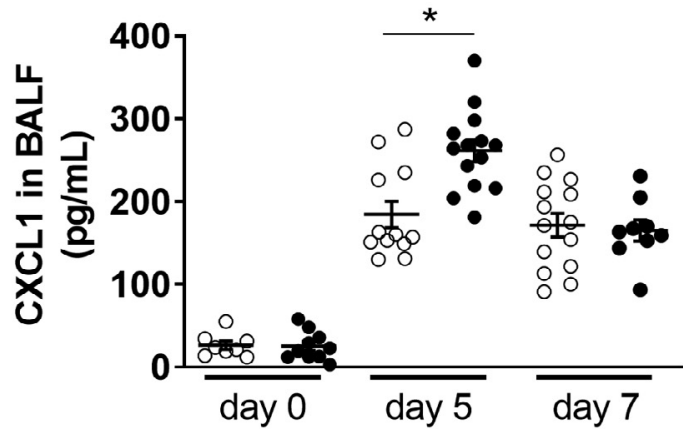

D

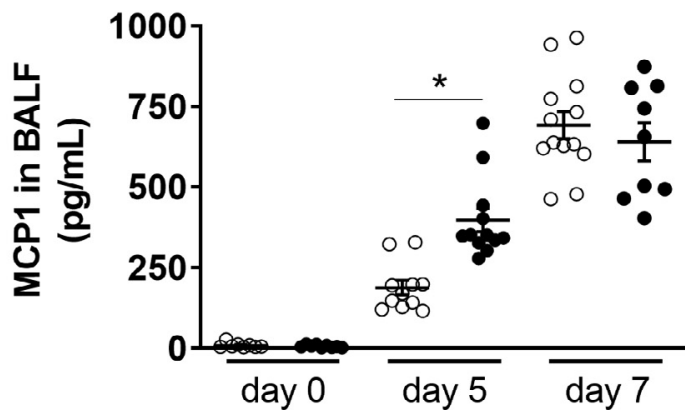

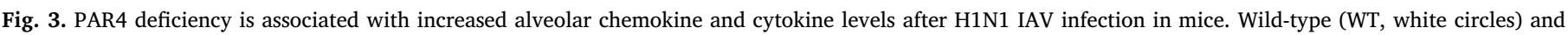

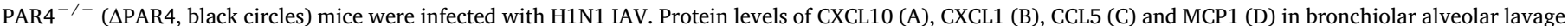
fluid (BALF) prior, 5 and 7 days after infection were analyzed by ELISA. Data (mean \pm SEM, N $>8$ ) were analyzed by 2 -way ANOVA. * P < 0.05 .

effect on the adaptive immune responses, we analyzed the IgG2a levels in serum before and 8 days p.i. We found that CVB3 infection increased the serum levels of IgG2a in both WT and $\triangle$ PAR4 mice (Fig. 2J). Interestingly, PAR4 deficiency was associated with significant lower levels of IgG2a 8 days p.i. compared to WT mice (Fig. 2J).

\subsection{PAR4 deficiency is associated with increased BALF cytokine and chemokine expression after H1N1 IAV infection of mice}

To investigate the effect of PAR4 deficiency in virus infection of the lung, we infected WT and $\triangle$ PAR4 mice with a mouse-adapted H1N1 IAV and collected BALF. Both genotypes had similar levels of CXCL10, CXCL1, CCL5 and MCP1 in the BALF prior to H1N1 IAV infection (Fig. 3). H1N1 IAV infection induced the expression of these inflammatory mediators. Interestingly, BALF from $\triangle$ PAR4 mice had significant higher levels of CXCL10, CXCL1, CCL5 and MCP1 5 days p.i. with H1N1 IAV compared to WT mice (Fig. 3). However, this differences between the genotypes were no longer observed at day 7 p.i. (Fig. 3).

\subsection{PAR4 deficiency leads to an early increase in cellular inflammation} into the lung after H1N1 IAV infection of mice

To quantify the amount of cellular inflammation, numbers of total white blood cells (WBC), neutrophils, lymphocytes and macrophages were measured before as well as 5 and 7 days after H1N1 IAV infection. Both genotypes exhibited similar numbers of these cell types in the BALF prior to H1N1 IAV infection (Fig. 4). As expected, H1N1 IAV infection led to an infiltration of cells into the lung and airspace of both genotypes as shown by the increased numbers of immune cells in the collected BALF 5 and 7 days p.i. (Fig. 4). Interestingly, $\triangle$ PAR4 mice exhibited significant more WBC, neutrophils, lymphocytes and monocytes in BALF 5 days after H1N1 IAV infection (Fig. 4). However, there were no longer any differences when the cellular content of BALF from
WT and $\triangle$ PAR4 was compared at day 7 p.i. (Fig. 4).

\subsection{PAR4 deficiency is associated with increased H1N1 IAV genome levels}

Virus genome levels in the lung were analyzed 5 days p.i. to investigate if PAR4 deficiency has an effect of H1N1 IAV titers. As expected from our observation with CVB3 infection, $\triangle$ PAR4 mice had significantly elevated H1N1 IAV genome levels in the lung compared to WT mice 5 days p.i. (Fig. 5A).

\subsection{PAR4 deficiency has a mild effect on alveolar bleeding after IAV infection}

Recently, we showed that H1N1 IAV infection leads to a mild bleeding in the lung of mice [22]. Furthermore, the extrinsic pathway of blood coagulation was required to limit alveolar hemorrhages and improve survival of H1N1 IAV infected mice [21,22]. Since PAR4 is the main thrombin receptor on murine platelets, we analyzed markers of bleeding in BALF collected prior and 5 and 7 days after H1N1 IAV infection of WT and $\triangle$ PAR4 mice. Prior to viral infection we found similar levels of red blood cells (RBC) in BALF of both genotypes (Fig. 5B). As expected, H1N1 IAV infection led to increased extravasation of RBC into the airspace of the lung in both WT and $\triangle$ PAR4. However, PAR4 deficiency was associated with higher numbers of RBC compared to WT mice 5 and 7 days after H1N1 IAV infection (Fig. 5B).

\subsection{PAR4 deficiency is associated with increased lung pathology after H1N1 IAV infection of mice}

H\&E stained lung sections showed that lungs of $\triangle$ PAR4 mice exhibited an increase in infiltrating cells in the airspace (bronchial inflammation) and increase in overall cellular inflammation within the lung (interstitial inflammation) compared to WT mice lungs 5 days after H1N1 IAV infection (Fig. 6). 


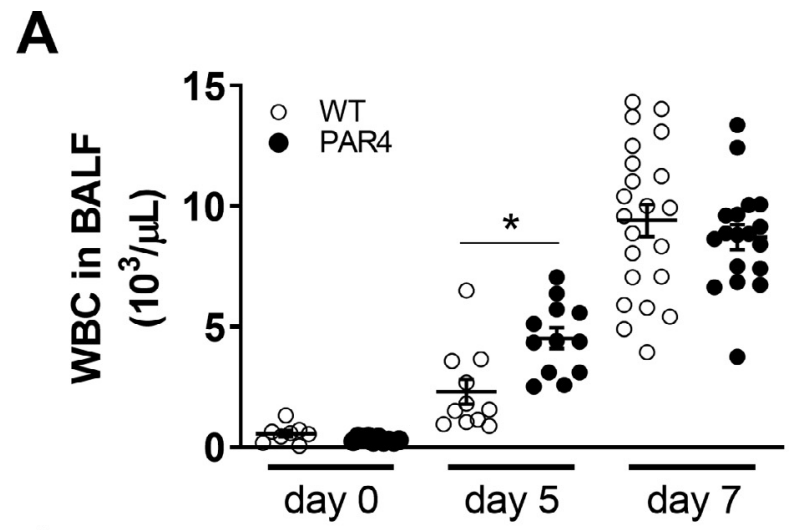

B
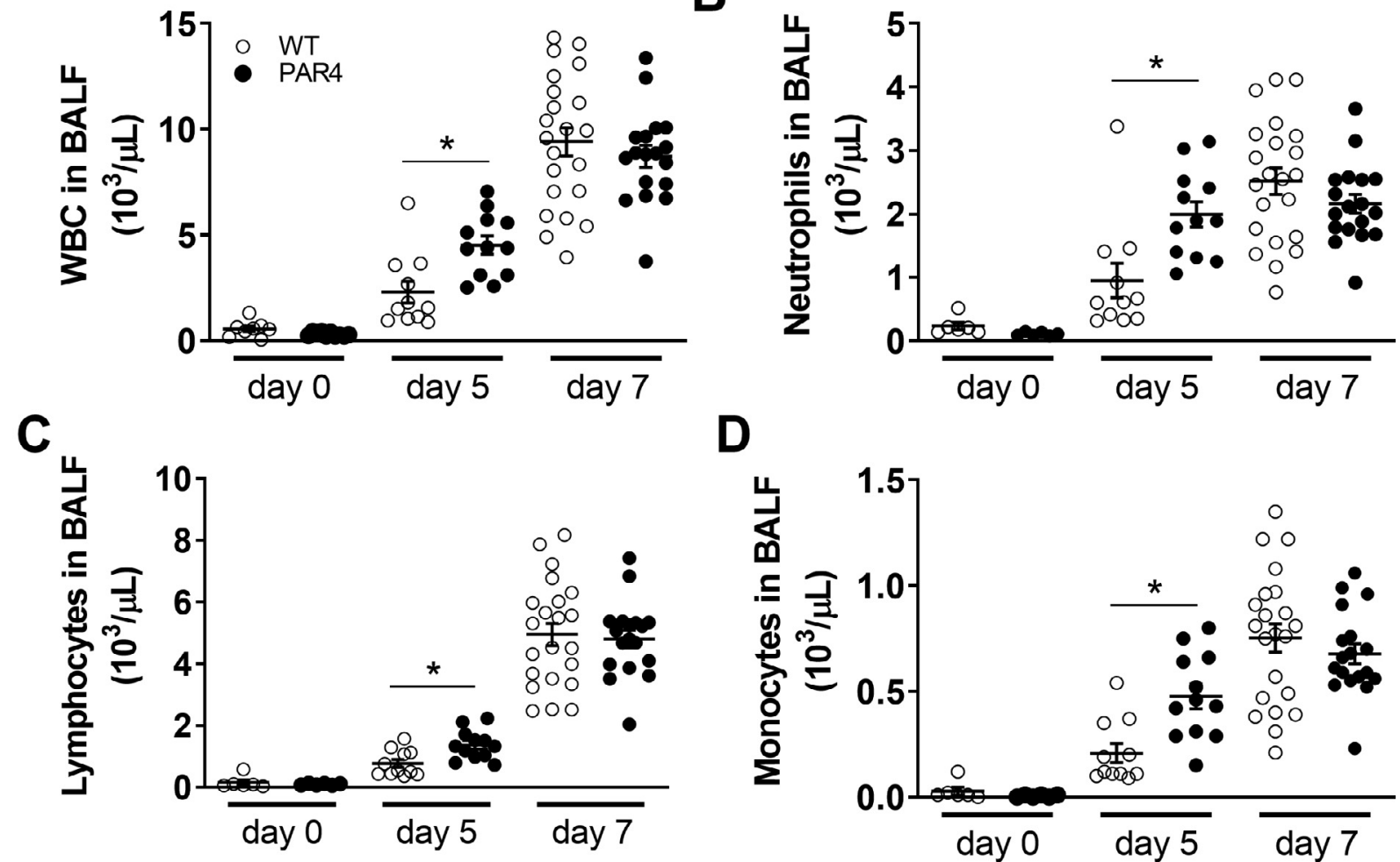

D

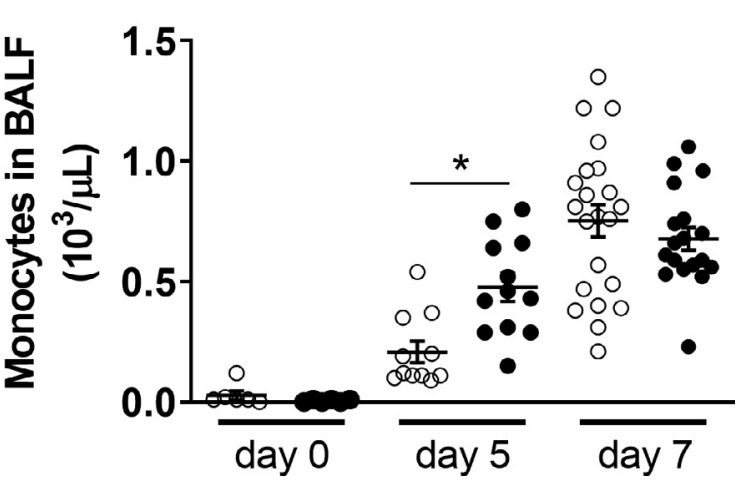

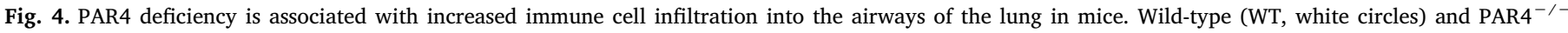

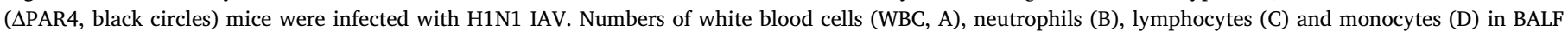
prior, 5 and 7 days after infection were quantified by HemoVet. Data (mean \pm SEM, N $>5$ ) were analyzed by 2 -way ANOVA. * $\mathrm{P}<0.05$.

\subsection{PAR4 deficiency is associated with increased IAV infection-induced} lung injury and mortality of mice

As expected, the increased inflammation resulted in more severe lung injury in $\triangle$ PAR4 mice compared to WT mice as measured by total protein levels in BALF 5 days after H1N1 IAV infection (Fig. 7A). $\triangle$ PAR4 and WT mice exhibited similar changes in body weights after infection (Fig. 7B). However, PAR4 deficiency was associated with a significantly increased mortality starting at day 8 p.i. compared to infected WT mice (Fig. 7C). The calculated hazard ratio (HR) for global PAR4 deficient mice was 2.53 compared to WT mice.

\subsection{Platelet inhibition protects mice from H1N1 IAV infection}

Since murine platelet PAR4 is needed for maximal platelet activation, we analyzed the effect of inhibition of thromboxane-mediated platelet activation by treating mice with ASA or inhibiting the plateletdependent ADP signaling by blocking the murine P2Y12 receptor with clopidogrel. ASA as well as clopidogrel-treated mice showed no difference in protein levels in BALF either before or 7 days after H1N1 IAV infection compared to vehicle treated control mice (Fig. 8A). However, clopidogrel and ASA treatment resulted in significant better recovery of body weights starting 8 and 9 days after IAV infection, respectively (Fig. 8B). Interestingly, only ASA treatment protected mice from H1N1
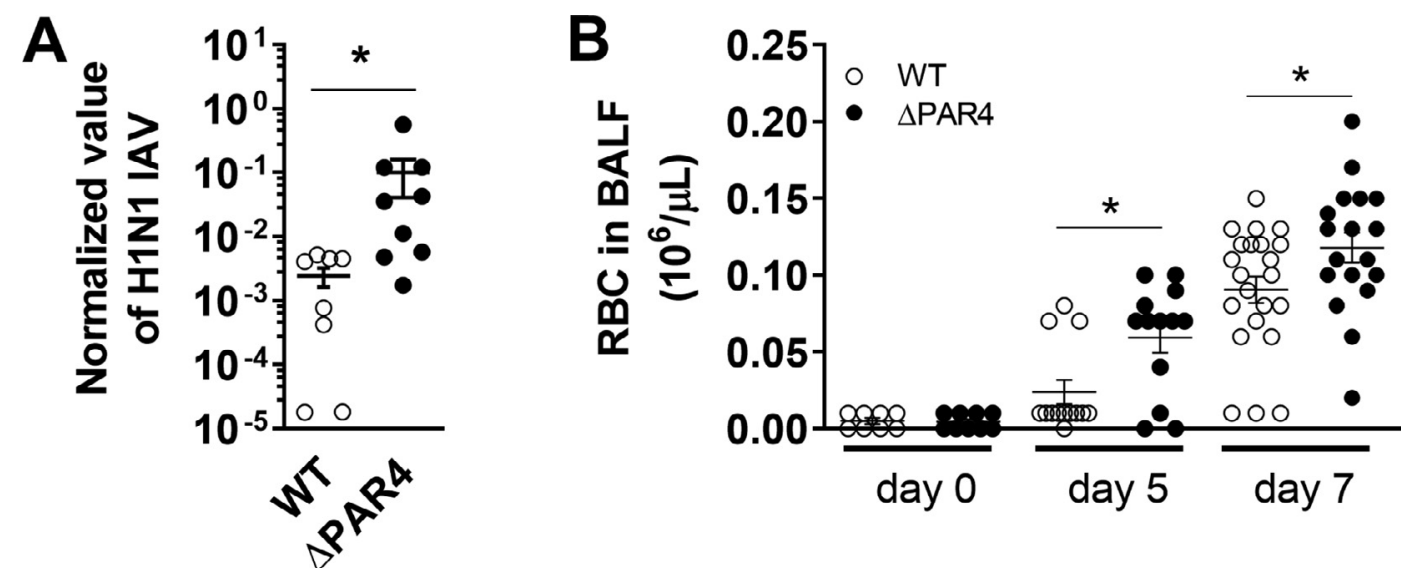

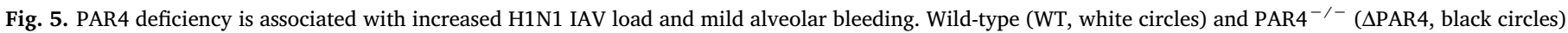

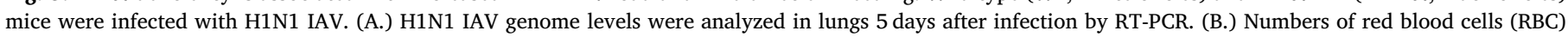

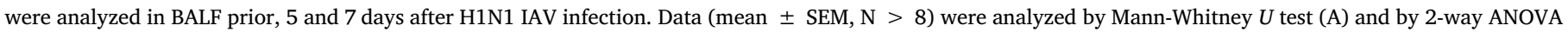
(B). ${ }^{*} \mathrm{P}<0.05$. 

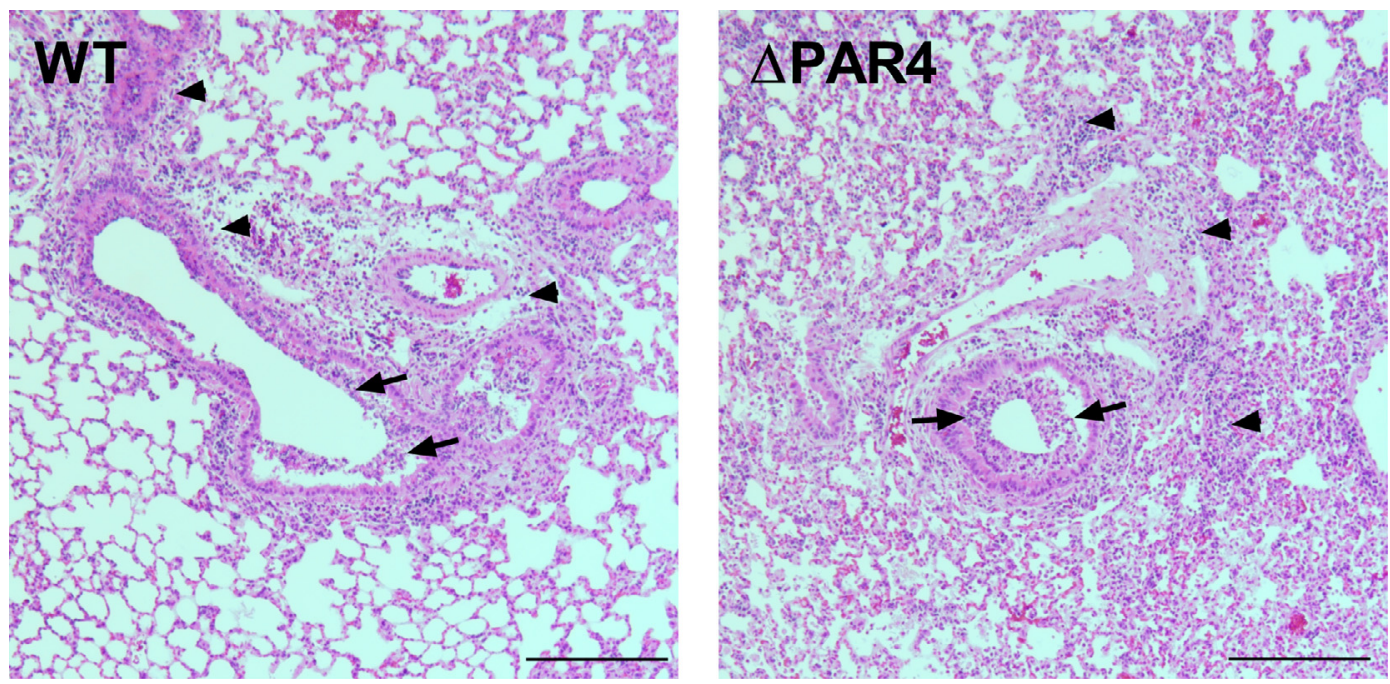

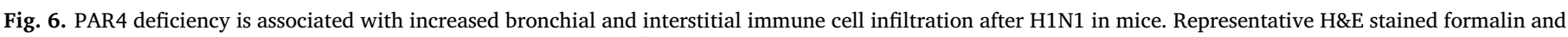

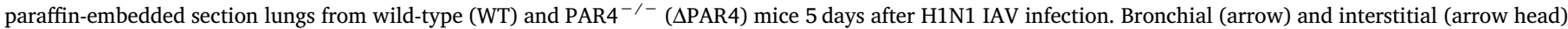
immune cell infiltration within the lung are indicated. Size bar $=500 \mu \mathrm{m}$.

IAV-induced death (Fig. 8C). The calculated HR for ASA compared to vehicle treatment is 0.137 whereas for clopidogrel compared to vehicle treatment is only 0.630 .

\section{Discussion}

In this study, we used global PAR4 deficient mice to investigate the role of PAR4 in two human disease relevant virus infection models CVB3 myocarditis and H1N1 IAV infection. We observed that PAR4 protects the heart and lung against CVB3 and H1N1 IAV infection, respectively. As previously shown for PAR1, we observed that PAR4 enhanced TLR3:IRF3-dependent IFN responses by increasing IFN $\beta$ and CXCL10 expression in vivo and in vitro. We think that PAR1 and PAR4 use a common pathway in positively increasing TLR3-dependent antiviral signaling possibly via enhancing p38 activation [8]. Interestingly, we found that while PAR4 enhances the TLR3:IRF3 pathway it dampens the TLR3:NFKB pathway leading to reduced expression of proinflammatory mediators, such as TNF $\alpha$, MCP1 and CXCL1. Our findings contribute to studies by us and others that thrombin-activatable PARs enhance antiviral TLR3 responses [8,11,12,29].

\subsection{PAR4 in CVB3 myocarditis}

We showed that thrombin as well as PAR1 contributes to an innate immune responses to CVB3 or the TLR3 agonist poly I:C $[8,11]$. This effect was due to PAR1-dependent TLR3 pathway enhancement on cardiac fibroblasts, cardiac myocytes, macrophages and splenocytes $[8,11]$. Interestingly, in CVB3 infection, platelets were identified as a major factor in limiting CVB3 myocarditis by reducing systemic viremia and enhancing immune cell function [30]. However, the study did not show that platelet activation was needed for the protection [30]. Important for our findings, PAR4 is not only expressed on murine platelets but it is also expressed by other cells, such as on CMs and immune cells $[3,17,27,31]$. Whether PAR4 is expressed by all kinds of fibroblast subtypes is still in question $[3,32,33]$. For instance, we found MEFs but not cardiac fibroblasts to express detectable PAR4 mRNA (data not shown) and are responsive to PAR4 stimulation. In this study, we showed that PAR4 activation enhances TLR3-dependent CXCL10 expression in BMMs and MEFs. CXCL10 mediates antiviral properties by recruiting NK cells to infected tissues [26]. Furthermore, we observed that PAR4 activation reduced TLR3-dependent NFKB responses, in at least WT MEFs and CMs, leading to reduced expression of MCP1 and
TNFa when TLR3 and PAR4 were co-stimulated. This PAR-dependent reduction in NFKB-dependent TLR3 responses was recently proposed by us for PAR1 [1]. Recruitment of macrophages into the heart was shown to be MCP1-dependent [34]. Furthermore, reduced cardiac MCP1 levels as well as cardiac macrophages numbers were associated with improved CVB3 myocarditis [35]. Our data point to a PAR4-dependent effect in reducing myocardial inflammation possibly by inhibiting the MCP1-macrophage axis.

We found increased CVB3 genomes in the heart of $\triangle$ PAR4 mice compared to WT mice after infection. The differences in viral genome levels appears to be due to the impaired anti-viral TLR3 immune responses in $\triangle$ PAR4 mice rather than to a PAR4-dependent effect on viral replication. We did not see any changes in viral replication when CMs were stimulated with PAR4 AP while thrombin reduced CVB3 genomes levels. This suggest that PAR1 rather than PAR4 reduced CVB3 replication in CMs.

\subsection{Effect of PAR4 stimulation on NK cell-like activity of splenocytes}

NK are important in limiting viral spreading, including CVB3, by killing virus infected cells [26]. In addition, thrombin activation was shown to increase NK cell-like activity in human peripheral blood lymphocytes [36]. In the past, we showed that PAR1 activation enhances NK cell-like cytotoxicity of mouse splenocytes [8]. In addition to PAR1, NK cells express also PAR4 [37]. We found that NK cell-like activity of splenocytes was increased in a PAR4-dependent way, suggesting that PAR4 directly influences NK cell function possibly via increase IL-2 release as shown for thrombin stimulation of human NK cells [36].

\subsection{PAR4 in adaptive immune responses in CVB3 myocarditis}

To analyze the possible effect of PAR4 on the adaptive immune system, we measured IgG responses after CVB3 infection in WT compared to $\triangle$ PAR4 mice. IgG2a titers increases after viral infections [38]. IgG in particular IgG2a were linked to increased viral elimination of CVB3 and other viruses $[28,39,40]$. Moreover, IgG2a expression was dependent to IFN $\gamma$-dependent signaling after virus infection $[39,40]$. IgG2a antibodies were shown to be more effective in activating complement, stimulating macrophages through the IgG Fc receptor and enhancing antibody-dependent cellular cytotoxicity [41,42]. While $\triangle$ PAR4 mice had a more pronounced CVB3 myocarditis 8 days after 

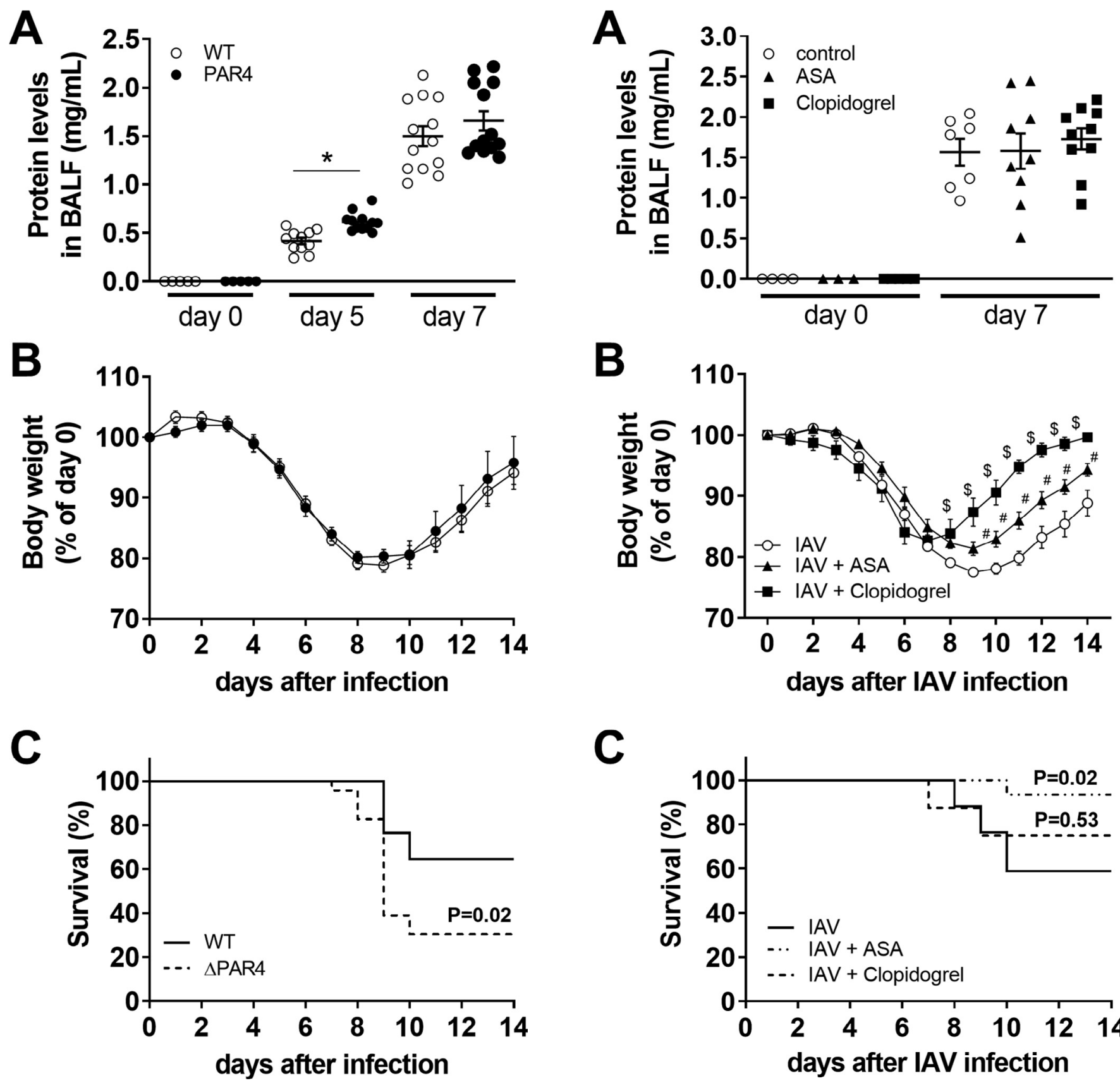

Fig. 7. PAR4 deficiency is associated with increased lung injury and mortality after IAV infection. Wild-type (WT, white circles) and PAR4 ${ }^{-/-}(\triangle \mathrm{PAR} 4$, black circles) mice were infected with H1N1 IAV. (A) Lung injury was quantified by total protein concentration in BALF prior, 5 and 7 days after infection. Changes in body weights (B) and calculated survival (C) over 14 days after infection ( $\mathrm{N}=17$ for $\mathrm{WT}$ and $\mathrm{N}=23$ for $\triangle \mathrm{PAR} 4$ ). Body weights before infection were set to $100 \%$. Data (mean $\pm \mathrm{SEM}$ ) and calculated survival were analyzed by 2 -way ANOVA (A and B) or log-rank test (C). * $\mathrm{P}<0.05$.

infection their serum IgG2a levels were significant lower compared to WT mice. This suggest that PAR4 plays a role in adaptive IgG2a immune responses at least to CVB3. Interestingly, it was shown that platelets enhance the total IgG response after CVB3 infection [30]. Whether the platelet dependent effect on IgG levels after CV3 infection and our observation was due to platelet PAR4 or independent of each other needs further investigation.

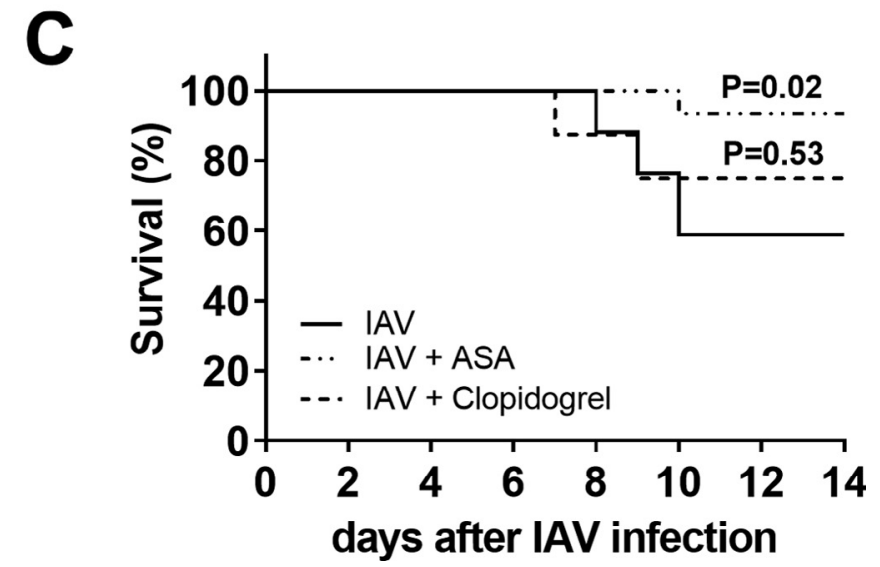

Fig. 8. Pharmacologic platelet inhibition mediates protection against H1N1 IAV infection in mice. Wild-type mice were treated with vehicle (control, white circles), acetylsalicylic acid (ASA, $0.4 \mathrm{mg} / \mathrm{mL}$, black triangles) or clopidogrel $(0.15 \mathrm{mg} / \mathrm{mL}$, black squares) in drinking water 3 days prior and after H1N1 IAV infection. A.) Protein levels in BALF of mice treated with vehicle, ASA or clopidogrel before and 7 days after H1N1 IAV infection. Changes in body weights (B) and calculated survival (C) over 14 days after H1N1 IAV infection (N = 17 for vehicle, $\mathrm{N}=15$ for ASA and $\mathrm{N}=8$ for clopidogrel). Body weights before infection were set to $100 \%$. Data (mean \pm SEM) and calculated survival were analyzed by 2 -way ANOVA (A, B) or log-rank test (C). ${ }^{*} \mathrm{P}<0.05$, \# P $<0.05$ IAV + ASA versus IAV (vehicle) alone, $\$ \mathrm{P}<0.05$ IAV + clopidogrel versus IAV (vehicle) alone.

\subsection{PAR4 in H1N1 IAV infection}

The seasonal flu is a reoccurring public health threat causing serious morbidity and mortality in the young and elderly population. Our study is in contrast to the findings by another study [18]. Here, we showed that a lack of PAR4 leads to a more pronounced H1N1 influenza A 
infection whereas Lê and colleagues [18] found that P4pal-10-dependent PAR4 inhibition improved H1N1 IAV infection in mice. Furthermore, they showed that exogenous PAR4 stimulation by intranasal application of PAR4 AP daily for the first 3 days after H1N1 IAV infection increased mortality in mice. However, the specificity of P4pal10 was not examined in PAR4 deficient mice. Furthermore, since the authors applied the PAR4 AP intranasally they locally overactivate PAR4 in the airspace which potentially does not occur during the normal course of H1N1 IAV infection. Furthermore, it was suggested that PARs exhibit a biased signaling pattern dependent on the agonist, such as proteolytic versus AP activation [43]. In our opinion using $\triangle$ PAR4 mice is a better approach to identify a potential role of PAR4 in an investigated disease setting.

The hyperinduction of proinflammatory cytokines also called cytokine storm caused by influenza virus infection is thought to be responsible for the infection associated complications and death. Importantly for H1N1 IAV infection, it was shown that a lack of TLR3:NFKB-dependent inflammation facilitated protection $[44,45]$. Our data suggests that PAR4 reduced the activation the TLR3:NFKB pathway in vitro and in vivo. This observation is novel since it was proposed that PAR4 stimulation alone induces a proinflammatory phenotype [46]. In support of our findings, Gomides et al. reported that PAR4 inhibition with tcY-NH2 increased lung CXCL1 expression in a carrageenan lung inflammation model [47]. The mechanism by which PAR4 activation reduces TLR3-dependent NFKB activation has not been elucidated. However, activated PAR4 interacts with $\beta$-arrestin2 [48], which has been shown to facilitates endothelial protein $C$ receptor-mediated inhibition of NFKB via PAR1 [49]. Moreover, $\beta$-arrestin2 was shown to inhibit TLR-dependent NFKB activation [50]. Further studies are required to determine if PAR4 affects $\beta$-arrestin2.

\subsection{Platelets in H1N1 IAV infections}

Several studies showed that platelets are activated during virus infections, including IAV $[1,18,30,51,52]$. Furthermore, use of different anti-platelet drugs was shown to reduce IAV infection in mice $[1,18,53]$. We found that H1N1 IAV infections of the lung is a hemostatic challenge and reduced activation of coagulation can lead to increased alveolar hemorrhages and death [21,22]. Furthermore, we showed that while thrombin inhibition increased alveolar hemorrhages it did not affect survival of H1N1 IAV infected mice [21]. Interestingly, we saw only mild signs of alveolar hemorrhages but no signs of impaired activation of coagulation (levels of thrombin-antithrombin complexes) in the BLAF after H1N1 IAV infection in $\triangle$ PAR4 mice compared to WT mice (data not shown). In addition, we confirmed data by others that platelet inhibition with clopidogrel as well as ASA reduced H1N1 IAV infection in mice $[18,53]$.

Our study suggest that our observation in PAR4 deficient mice was thrombin-PAR4 as well as platelet PAR4-independent since we showed that thrombin inhibition in H1N1 IAV infection has no influence on diseases progression in mice [21] while platelet inhibition is protective as shown by us and others $[18,53]$.

\subsection{PAR4 inhibition in H1N1 IAV infection}

The inhibition of the major thrombin receptor on human platelets, PAR1, was initially proposed to be beneficial due to reduce pathologic platelet activation [6]. However, clinical trials showed later the importance of PAR1 on endothelial cells in reducing the reoccurrence of stroke [6]. This reduces the potential application of the FDA-approved PAR1 inhibitor vorapaxar. Therefore, PAR4 became a new target to reduced platelet activation and PAR4 antagonists have been developed as potential antiplatelet drugs $[13,16]$. Unfortunately, there is limited data on the role of PAR4 in cardiovascular diseases or vessel integrity [1,3,54-56]. Most studies used human or WT rodent platelets for in vitro as well as WT mice or rats for in vivo studies in combination with PAR4 inhibitors or activators $[18,52]$. However, as with all inhibitors there are concerns with specificity of these agents. Most of the studies lack control experiments to show that the compounds act via a PAR4-dependent mechanism. In addition, some of the PAR4 inhibitors were shown to act as biased agonists in vitro [57]. Importantly, various PAR activators and inhibitors showed PAR-independent actions [1,57-61]. For instance, the pepducin P4pal-10 was thought to block only PAR4 signaling [62]. However, it was shown that P4pal-10 inhibits other Gaq-coupled receptors, including PAR1, M3 muscarinic acetylcholine receptor, $\mathrm{H} 1$ histamine receptor and thromboxane $\mathrm{A} 2$ receptor $[60,61]$. The opposing results by Lê et al. [18] that PAR4 inhibition is protective in H1N1 IAV infection could therefore be explained by an off-target effect of P4pal-10 blocking the thromboxane A2 receptor [61] mimicking an ASA treatment leading to improved survival after H1N1 IAV infection.

In conclusion, our data suggest that platelet-independent PAR4 mediates protective signaling possibly on resident and immune cells to limit certain RNA virus infections. We do not think our observation was due to compensation by other PARs since a recent study showed that PAR4 deficiency does not influence the expression of other PARs [17]. Platelet PAR4 was identified as drug-able target to reduce thrombotic events. However, our data indicate that PAR4 is needed for effective anti-viral responses. Our findings point to a potential risk that the use of a PAR4 antagonists could lead to an increased incident and severity of certain virus infection in patients. This risk should be investigated in future studies.

\section{Study limitations}

We cannot finally exclude the potential contribution of PAR4 on platelets in H1N1 IAV or CVB3 infection. The use of a conditional PAR4 knock-out mouse will help to analyze the cell-specific contribution of PAR4 in various diseases models including H1N1 IAV infection and CVB3 myocarditis. Importantly, additional studies with PAR4 antagonists in WT and $\triangle$ PAR4 mice are needed.

\section{Grant support}

The study was supported by the NIH to N. Mackman (HL119523) and S. Antoniak (HL142799).

\section{Declaration of Competing Interest}

None.

\section{Acknowledgment}

We want to thank Ying Zhang for excellent technical assistance.

\section{References}

[1] S. Antoniak, N. Mackman, Multiple roles of the coagulation protease cascade during virus infection, Blood 123 (17) (2014) 2605-2613.

[2] S.R. Coughlin, Thrombin signalling and protease-activated receptors, Nature 407 (6801) (2000) 258-264.

[3] S. Antoniak, N. Mackman, Coagulation, protease-activated receptors, and viral myocarditis, J Cardiovasc. Transl. Res. 7 (2) (2014) 203-211.

[4] M.L. Kahn, et al., A dual thrombin receptor system for platelet activation, Nature 394 (6694) (1998) 690-694.

[5] G.R. Sambrano, et al., Role of thrombin signalling in platelets in haemostasis and thrombosis, Nature 413 (6851) (2001) 74-78.

[6] S. Lam, T. Tran, Vorapaxar: a protease-activated receptor antagonist for the prevention of thrombotic events, Cardiol Rev 23 (5) (2015) 261-267.

[7] A. Weithauser, et al., Protease-activated receptor 2 regulates the Innate Immune Response to Viral Infection in a CVB3-induced Myocarditis, J. Am. Coll. Cardiol. 62 (19) (2013) 1737-1745.

[8] S. Antoniak, et al., PAR-1 contributes to the innate immune response during viral infection, J. Clin. Invest. 123 (3) (2013) 1310-1322.

[9] Q.M. Nhu, et al., Novel signaling interactions between proteinase-activated receptor 2 and Toll-like receptors in vitro and in vivo, Mucosal. Immunol. 3 (1) (2010) 
29-39.

[10] K. Khoufache, et al., PAR1 contributes to influenza A virus pathogenicity in mice, J Clin Invest 123 (1) (2013) 206-214.

[11] S. Antoniak, et al., Protease-Activated Receptor 1 Enhances Poly I: C Induction of the Antiviral Response in Macrophages and Mice, J. Innate Immun. 9 (2) (2017) 181-192.

[12] J. Zhang, et al., Innate generation of thrombin and intracellular oxidants in airway epithelium by allergen Der p 1, J. Allergy Clin. Immunol. 138 (4) (2016) 1224-1227.

[13] W. Bergmeier, et al., Advances in clinical and basic science of coagulation: illustrated abstracts of the 9th Chapel Hill symposium on hemostasis, Res. Pract. Thromb. Haemost. 2 (3) (2018) 407-428.

[14] K. Khoufache, et al., Protective role for protease-activated receptor-2 against influenza virus pathogenesis via an IFN-gamma-dependent pathway, J. Immunol. 182 (12) (2009) 7795-7802.

[15] G. Rwibasira Rudinga, G.J. Khan, Y. Kong, Protease-activated receptor 4 (PAR4): a promising target for antiplatelet therapy, Int. J. Mol. Sci. 19 (2) (2018) E573.

[16] P.C. Wong, et al., Blockade of protease-activated receptor-4 (PAR4) provides robust antithrombotic activity with low bleeding, Sci. Transl. Med. 9 (371) (2017) eaaf5294.

[17] M.A. Kolpakov, et al., Protease-activated receptor 4 deficiency offers cardioprotection after acute ischemia reperfusion injury, J. Mol. Cell. Cardiol. 90 (2016) $21-29$.

[18] V.B. Lê, et al., Platelet activation and aggregation promote lung inflammation and influenza virus pathogenesis, Am. J. Respir. Crit. Care Med. 191 (7) (2015) 804-819.

[19] A.G. Smith, et al., Diet-induced obese mice have increased mortality and altered immune responses when infected with influenza virus, J. Nutr. 137 (5) (2007) 1236-1243.

[20] S. Antoniak, et al., Protease-activated receptor 1 activation enhances doxorubicininduced cardiotoxicity, J. Mol. Cell. Cardiol. 122 (2018) 80-87.

[21] K. Tatsumi, et al., Anticoagulation increases alveolar hemorrhage in mice infected with influenza A, Physiol. Rep. 4 (24) (2016) e13071.

[22] S. Antoniak, et al., Tissue factor deficiency increases alveolar hemorrhage and death in influenza A virus-infected mice, J. Thromb. Haemost. 14 (6) (2016) 1238-1248.

[23] N. Joshi, et al., Inhibition of PAR-4 and P2Y12 receptor-mediated platelet activation produces distinct hepatic pathologies in experimental xenobiotic-induced cholestatic liver disease, Toxicology 365 (2016) 9-16.

[24] A. Lauer, et al., Antiplatelet pretreatment does not increase hematoma volume in experimental intracerebral hemorrhage, J. Cereb. Blood Flow Metab. 31 (8) (2011) $1736-1742$.

[25] K. Klingel, et al., Visualization of enteroviral replication in myocardial tissue by ultrastructural in situ hybridization: identification of target cells and cytopathic effects, Lab. Invest. 78 (10) (1998) 1227-1237.

[26] J. Yuan, et al., CXCL10 inhibits viral replication through recruitment of natural killer cells in coxsackievirus B3-induced myocarditis, Circ. Res. 104 (5) (2009) 628-638.

[27] A. Rullier, et al., Expression of protease-activated receptors and tissue factor in human liver, Virchows Arch. 448 (1) (2006) 46-51.

[28] C. Leipner, et al., Coxsackievirus B3-induced myocarditis: differences in the immune response of C57BL/6 and Balb/c mice, Med. Microbiol. Immunol. 193 (2-3) (2004) 141-147.

[29] S. Antoniak, The coagulation system in host defense, Res. Pract. Thromb. Haemost. 2 (3) (2018) 549-557.

[30] S. Negrotto, et al., Platelets interact with Coxsackieviruses B and have a critical role in the pathogenesis of virus-induced myocarditis, J. Thromb. Haemost. 13 (2) (2015) 271-282.

[31] A. Sabri, et al., Mechanisms of protease-activated receptor-4 actions in cardiomyocytes. Role of Src tyrosine kinase, J. Biol. Chem. 278 (13) (2003) 11714-11720.

[32] S. Shimizu, et al., Thrombin and activated coagulation factor X stimulate the release of cytokines and fibronectin from nasal polyp fibroblasts via protease-activated receptors, Am J Rhinol Allergy 31 (1) (2017) 13-18.

[33] S. Kleeschulte, et al., Evidence for functional PAR-4 thrombin receptor expression in cardiac fibroblasts and its regulation by high glucose: PAR-4 in cardiac fibroblasts, Int. J. Cardiol. 252 (2018) 163-166.

[34] P.E. Kolattukudy, et al., Myocarditis induced by targeted expression of the MCP-1 gene in murine cardiac muscle, Am. J. Pathol. 152 (1) (1998) 101-111.

[35] C. Jaquenod De Giusti, et al., Macrophages and galectin 3 play critical roles in CVB3-induced murine acute myocarditis and chronic fibrosis, J. Mol. Cell. Cardiol. 85 (2015) 58-70.
[36] A. Naldini, D.H. Carney, Thrombin modulation of natural killer activity in human peripheral lymphocytes, Cell. Immunol. 172 (1) (1996) 35-42.

[37] S. Shrivastava, J.H. McVey, A. Dorling, The interface between coagulation and immunity, Am. J. Transplant. 7 (3) (2007) 499-506.

[38] J.P. Coutelier, et al., IgG2a restriction of murine antibodies elicited by viral infections, J. Exp. Med. 165 (1) (1987) 64-69.

[39] V.K. Baxter, D.E. Griffin, Interferon gamma modulation of disease manifestation and the local antibody response to alphavirus encephalomyelitis, J. Gen. Virol. 97 (11) (2016) 2908-2925.

[40] K. Rubtsova, et al., T-box transcription factor T-bet, a key player in a unique type of B-cell activation essential for effective viral clearance, Proc. Natl. Acad. Sci. USA 110 (34) (2013) E3216-E3224.

[41] W.J. Johnson, et al., Destructive interactions between murine macrophages, tumor cells, and antibodies of the IgG2a isotype, Adv. Exp. Med. Biol. 184 (1985) 75-80.

[42] T.J. Kipps, et al., Importance of immunoglobulin isotype in human antibody-dependent, cell-mediated cytotoxicity directed by murine monoclonal antibodies, J. Exp. Med. 161 (1) (1985) 1-17.

[43] P. Zhao, M. Metcalf, N.W. Bunnett, Biased signaling of protease-activated receptors, Front. Endocrinol. (Lausanne) 5 (2014) 67.

[44] R. Le Goffic, et al., Detrimental contribution of the Toll-like receptor (TLR)3 to influenza A virus-induced acute pneumonia, PLoS Pathog. 2 (6) (2006) e53.

[45] C. Huo, et al., Lethal influenza A virus preferentially activates TLR3 and triggers a severe inflammatory response, Virus Res. 257 (2018) 102-112.

[46] Z. Suo, et al., Persistent protease-activated receptor 4 signaling mediates thrombininduced microglial activation, J. Biol. Chem. 278 (33) (2003) 31177-31183.

[47] L.F. Gomides, et al., Blockade of proteinase-activated receptor 4 inhibits neutrophil recruitment in experimental inflammation in mice, Inflamm. Res. 63 (11) (2014) 935-941.

[48] D. Li, et al., Arrestin-2 differentially regulates PAR4 and ADP receptor signaling in platelets, J. Biol. Chem. 286 (5) (2011) 3805-3814.

[49] R.V. Roy, et al., Occupancy of human EPCR by protein C induces beta-arrestin-2 biased PAR1 signaling by both APC and thrombin, Blood 128 (14) (2016) 1884-1893.

[50] N. Parameswaran, et al., Arrestin-2 and G protein-coupled receptor kinase 5 interact with NFkappaB1 p105 and negatively regulate lipopolysaccharide-stimulated ERK1/2 activation in macrophages, J. Biol. Chem. 281 (45) (2006) 34159-34170.

[51] M.T. Rondina, et al., In vivo platelet activation in critically ill patients with primary 2009 influenza A(H1N1), Chest 141 (6) (2012) 1490-1495.

[52] E. Boilard, et al., Influenza virus H1N1 activates platelets through FcgammaRIIA signaling and thrombin generation, Blood 123 (18) (2014) 2854-2863.

[53] M.G. Sugiyama, et al., Influenza virus infection induces platelet-endothelial adhesion which contributes to lung injury, J. Virol. 90 (4) (2016) 1812-1823.

[54] S. Antoniak, E. Sparkenbaugh, R. Pawlinski, Tissue factor, protease activated receptors and pathologic heart remodelling, Thromb. Haemost. 112 (5) (2014) 893-900.

[55] M. Fujiwara, et al., Activation of PAR4 induces a distinct actin fiber formation via p38 MAPK in human lung endothelial cells, J. Histochem. Cytochem. 53 (9) (2005) 1121-1129.

[56] J.R. Hamilton, A.G. Frauman, T.M. Cocks, Increased expression of protease-activated receptor-2 (PAR2) and PAR4 in human coronary artery by inflammatory stimuli unveils endothelium-dependent relaxations to PAR2 and PAR4 agonists, Circ. Res. 89 (1) (2001) 92-98.

[57] M.D. Hollenberg, et al., Proteinase-activated receptor-4: evaluation of tethered ligand-derived peptides as probes for receptor function and as inflammatory agonists in vivo, Br. J. Pharmacol. 143 (4) (2004) 443-454.

[58] C. Di Serio, et al., Protease-activated receptor 1-selective antagonist SCH79797 inhibits cell proliferation and induces apoptosis by a protease-activated receptor 1independent mechanism, Basic Clin. Pharmacol. Toxicol. 101 (1) (2007) 63-69.

[59] R.J. Betts, T.S. Mann, P.J. Henry, Inhibitory influence of the hexapeptidic sequence SLIGRL on influenza A virus infection in mice, J. Pharmacol. Exp. Ther. 343 (3) (2012) 725-735.

[60] R. Carr 3rdet al., Interdicting Gq Activation in Airway Disease by ReceptorDependent and Receptor-Independent Mechanisms, Mol. Pharmacol. 89 (1) (2016) 94-104.

[61] J.J. Stampfuss, K. Schror, A.A. Weber, Inhibition of platelet thromboxane receptor function by a thrombin receptor-targeted pepducin, Nat Med 9 (12) (2003) 1447 author reply 1447-8.

[62] L. Covic, et al., Pepducin-based intervention of thrombin-receptor signaling and systemic platelet activation, Nat. Med. 8 (10) (2002) 1161-1165. 